\title{
Hippotherapy in Children with Developmental Delays: Physical Function and Psychological Benefits
}

\author{
Freda Thompson, Caroline J. Ketcham*, Eric E. Hall \\ Department of Exercise Science, Elon University, Elon, USA \\ Email: ${ }^{*}$ cketcham@elon.edu
}

Received 8 February 2014; revised 9 March 2014; accepted 14 March 2014

Copyright (C) 2014 by authors and Scientific Research Publishing Inc.

This work is licensed under the Creative Commons Attribution International License (CC BY). http://creativecommons.org/licenses/by/4.0/

(c) (i) Open Access

\begin{abstract}
Hippotherapy, or horse therapy, is an innovative form of physical therapy that involves the use of a horse as a therapeutic tool in order to effectively challenge a rider's core muscles. The rhythm of the horse's gait continuously engages the client's balance and posture, while the supervising physical therapist seeks to improve the client's overall coordination and fine motor skills through various activities. This combination of movement and therapist-directed activity can have significant impacts on the client's postural control and physical function. Eight children between the ages of 2 - 12 years that had been previously diagnosed with a variety of different developmental delays or disorders participated in 8 - 12 weeks of hippotherapy. Subjects were tested before, during, and at the completion of their therapy. The physical function measures included a timed level and unlevel walking test, a coordination-based reach test, and were scored using the Gainesville Riding through Equine Assisted Therapy (G.R.E.A.T.) Postural Scale to measure quality of posture. Psychological changes were also evaluated based on the physical therapists' notes and on parent questionnaires given pre- and post-study. Results indicated that $62.5 \%(5 / 8)$ of the subjects improved in their Postural Scale score, 87.5\% (7/8) decreased their time to complete the level and unlevel distances, and 50\% (4/8) of the subjects showed better coordination-based reach results from pre- to post-. Physical therapists' notes and preliminary parent report analyses suggest improvements in subjects' self-esteem and overall quality of life. These results indicate that hippotherapy sessions are a very effective way to improve physical function, specifically postural control, strength, and coordination in children who have been diagnosed with any type of developmental delay or disorder. Additionally children showed improvements in confidence, self-esteem, and quality of life as observed by their therapist and measured by the parent questionnaires, which may be directly related to the interaction with the horse and therapists or indirectly related to the improvements in motor function. This research has implications for the benefits of hippotherapy for the pediatric population for both function and quality of life.
\end{abstract}

\footnotetext{
"Corresponding author.
}

How to cite this paper: Thompson, F., Ketcham, C. J., \& Hall, E. E. (2014). Hippotherapy in Children with Developmental Delays: Physical Function and Psychological Benefits. Advances in Physical Education, 4, 60-69.

http://dx.doi.org/10.4236/ape.2014.42009 


\section{Keywords}

\section{Postural Control, Function, Quality of Life}

\section{Introduction}

The prevalence of children with developmental disabilities that may cause lifelong issues has increased 15\% (10 million children) between 2006-2008 (Boyle et al., 2001). The number of children who participate in therapeutic intervention compared to the number who qualify and could benefit from it is on the order of $2 \%$ (Rosenberg, Zhang, \& Robinson, 2008). That means there are millions of children who are not getting early intervention that could benefit their development and future. Therefore finding therapeutic interventions that improve function, are enjoyable to children and are accessible is important. Additionally, finding intervention modalities which addresses, physical (fine and gross motor), as well as social and emotional development can have even greater impact for the time and money spent. Interaction with animals has been shown to benefit function. In a metaanalysis animal assisted therapy, Nimer \& Lundahl (2007) was associated with moderate effect sizes showing improvements in Autism-spectrum symptoms, medical difficulties, behavioral problems and emotional wellbeing. Characteristics of participants did not produce differential outcomes suggesting that animal assisted therapy could result in additive effects of typical therapeutic intervention. Hippotherapy is a newer type of therapy that utilizes horses in conjunction with physical, occupational and speech-language therapy that has been reported to improve motivation, attention, and therefore quality of therapeutic time (Macauley \& Guiterrez, 2004).

Horseback riding is typically viewed as an extracurricular activity to the general public. It is an enjoyable, recreational sport where a rider is able to bond with the animal and successfully master various equine skills. However, most individuals are not aware of the therapeutic benefits that horseback riding can have on special populations, particularly populations where motor coordination deficits impact social, emotional, and academic aspects of a child's life (i.e. Cerebral Palsy, genetic disorders, Down Syndrome, and developmental delays; Taylor et al., 2009). Hippotherapy, or horse therapy, has just begun to receive attention in the world of physical, occupational, and speech therapy (Hammer et al., 2005). It involves the use of the horse as a therapeutic tool by engaging the rider's core muscles; the movement produced by the horse's gait forces the individuals to use their postural control in order to keep themselves upright (Shurtleff, Standeven, \& Engsberg, 2009). These improvements in postural control have been found in a number of the special populations mentioned and serve to reiterate the idea that this version of therapy is beneficial for these individuals. Along with improvements in motor and postural control, hippotherapy research also suggests that the social and emotional interactions can result in increases in quality of life and self-esteem (Bass, Duchowny, \& Llabre, 2009). Thus, this rehabilitative strategy is ideal for various special populations that would benefit from strengthening their postural control as well as building social interactions in an unconventional way.

In order to effectively and safely use this type of therapy within special populations, a physical or occupational therapist must be present at all times in order to lead the hippotherapy session and to instruct the rider to participate in the therapy session. Although the movement of the horse is the primary way to obtain physical function improvements, the activities that the rider performs also help to improve overall coordination and fine motor skills (Cantu, 2005). While the client is on horseback it is important that two individuals be positioned on either side of the horse to provide support and encouragement to the rider. These individuals, known as side walkers, must ensure that the client's safety is not compromised at any point by stabilizing the rider and following any additional instructions given by the therapist. The final member of the hippotherapy team is responsible for guiding the horse and making sure that the appropriate movements are instigated. Within a hippotherapy session, the therapist will request that the horse be led in specific patterns and at various speeds in order to guarantee that the rider's core is being challenged in all planes and movements (Hammer et al., 2005).

Despite the fact that horses have been seen as helpers for humans for several centuries, the rehabilitative component of horseback riding was not recognized until an Olympic medalist used it as her sole treatment for a muscle disorder (Meregillano, 2004). Since this first use of hippotherapy, this subject has intrigued many individuals. Many physicians and therapists now encourage the use of animal therapy for their patients because of the apparent psychological benefits that most kids have when in contact with these animals (Meregillano, 2004). 
By continuously placing the individual in contact with the horse in a comfortable setting, this therapy may effectively improve their quality of life and self-esteem.

What has been less objectively quantified is how this form of therapy helps motor components of development. Coordination of the musculoskeletal system is an integral part of balance, mobility and independence. Furthermore, coordination and control difficulties can have implications on psychological and cognitive development of individuals (Taylor et al., 2009). Therapeutic interventions, such as hippotherapy, are needed in order to enhance normal development and provide rehabilitation, to improve coordination and quality of life.

Movement scientists are specifically interested in the children's motor and postural control in order to address the coordination issues that play a role in the individuals' quality of life. One specific population, individuals with cerebral palsy, has been shown to have consistent positive motor outcomes following hippotherapy interventions (Sterba \& Warner, 2007). Cerebral palsy, delayed or impaired brain development, is comprised of a number of disorders, involving disabilities in the development of movement, as well as posture (Sterba \& Warner, 2007). Hippotherapy patients typically show improvements in motor outcomes because a horse's gait closely resembles that of a human's normal walking pattern; therefore, an individual who is unable to walk independently is able to assimilate this pattern via the gait of the horse (Zadnikar \& Kastrin, 2011; McGee \& Reese, 2009; Bertoti, 1988; Winchester, Kendall, Peters, Sears, \& Winkley, 2002). The horse’s movement induces three different responses in the rider: lateral pelvic tilt, anterior/posterior pelvic tilt, and pelvic rotation. All three of the responses when combined together incorporate the movements that would be made of the person's own accord if they were able to walk independently. It is estimated by therapists that the rhythmic 4-beat gait of the horse relates to at least 100 steps per minute and an average of 3000 steps for each session. The repetition of this movement throughout the session engages the riders' balance and posture, thereby improving their muscle tone and resulting in improved balance and coordination. Molloy, Dietrich and Bhattacharya (2003) conducted a study in which the authors were trying to compare postural control between children with Autism Spectrum Disorder and children with normal development. By testing sway patterns in both populations they found that the autistic individuals did have greater deficits in postural control than the other children. This specific disorder has received a great deal of research in regards to the social interaction aspects of the disorder, but relatively little studies have actually looked at the implications of the motor deficits found in this population (Molloy, Dietrich, \& Bhattacharya, 2003).

The purpose of this particular study is to examine the physical function (posture, strength, and coordination) and psychological (quality of life and self-esteem) benefits of a hippotherapy program in children with developmental delays. It is hypothesized that hippotherapy will improve physical function aspects such as postural control (i.e. posture, strength, and coordination) in individuals with developmental delays. It is also expected that quality of life and self-esteem of the individual will improve during the extent of their participation in hippotherapy sessions because of the additive benefits of animal assisted therapy.

\section{Methods}

\subsection{Participants}

The participants for this study were recruited from a physical therapy center that utilizes hippotherapy as a therapeutic intervention. Children came from a variety of different demographic backgrounds and were diagnosed with a disorder, involving developmental delays and/or motor deficits. Individuals were excluded from the study if they were unable to complete modified versions of the physical function measures or if they did not have proper understanding of the tasks. Eight participants between the ages of 2 - 12 years (6 males, 2 females) participated in this study (Table 1). Parents of the subjects were given Institutional Review Board approved letters describing the study, and they willingly signed consent forms for their child to participate in the current study. All subjects and parents understood that they could refrain from participation at any time, and they would receive a gift card for time compensation at the completion of the study.

\subsection{Protocol}

Participants of the study completed their hippotherapy sessions in Bethlehem, North Carolina at the Foothills Equestrian Center. Two physical therapists provided assistance throughout the 12-week program. The participants 
Table 1. Participant demographics and implications.

\begin{tabular}{|c|c|c|c|}
\hline Subject & Age/Gender & Diagnosis & Implications \\
\hline 1 & 3-Male & L side affected CVA & Cast on L leg \\
\hline 2 & 4-Female & Mild spastic CP, R-hemiparesis, hydrocephalus & Braces on both legs, use of walker as assistive device \\
\hline 3 & 5-Female & R leg amputee & Prosthetic leg on R side below knee joint \\
\hline 4 & 5-Male & Myotonic Dystrophy & Poor muscle tone, low motivation \\
\hline 5 & 10-Male & Mowat Wilson Syndrome, Epilepsy & Unable to speak, Vagus Nerve Stimulator to stop seizures \\
\hline 6 & 11-Male & $\mathrm{CP}$, developmental delays & Wheelchair bound, unable to speak, very high muscle tone \\
\hline 7 & 2-Male & $\mathrm{CP}$, developmental delays & $\begin{array}{l}\text { Communication difficulties, very young, } \\
\text { first experience with HPOT }\end{array}$ \\
\hline 8 & 3-Male & Motor apraxia, developmental delays, strabismus & Requires pediatric eye glasses, first experience with HPOT \\
\hline
\end{tabular}

attended a hippotherapy session once a week for forty-five minutes. This is a typical session of hippotherapy and comparable to the amount of physical therapy a child gets per week. During the time that the children were riding the horse, the therapists were responsible for engaging the children in various motor and sensory tasks to help the children activate and strengthen their core trunk muscles.

The physical function aspects of the study were taken pre-and post-hippotherapy intervention. Measurements included simple, objective tests described below that required core strength and coordination to complete the task. Participants were asked to complete at least two trials of each measure, and the best performances were recorded for each time frame. We kept the best performance because our goal was to assess ability not consistency of ability. The psychological components of this study were evaluated by having the children's parents fill out questionnaires addressing their quality of life and self-esteem. Such measures were completed before the study and after the final session to determine what, if any, improvements in psychological well-being occurred.

\subsection{Physical Function Measures}

Physical function tests were used to assess the participants' postural control, strength, and coordination. These tests were chosen by the supervising physical therapist because they were appropriate for the understanding levels of the pediatric subjects, and they reiterate activities that the therapists incorporate into their therapies. These measures were taken at two different points during the study: pre- and post-. Multiple trials were taken at each time, and the best score was recorded.

\subsubsection{G.R.E.A.T. Postural Scale}

The Gainesville Riding through Equine Assisted Therapy (G.R.E.A.T.) Postural Scale is an excellent indicator of postural control and balance and includes five specific areas of evaluation: head/cervical spine, shoulder/thoracic spine, pelvis/lumbar spine, hip angle, and knee flexion/heel orientation (Frease, 1996). Photos were taken from a lateral view while the subjects remained in a stable sitting position looking forward. One G.R.E.A.T. trained physical therapist analyzed the photos from each time frame and scored the photo according to the provided scale (Figure 1). This scoring was completed at the conclusion of the 12 weeks and the therapist did not know which was before or after and scored each individually. Each of the areas are added together for a total score which indicates how close the client is to appropriate body alignment. A total score of 0 indicates excellent posture, meaning that any scores above 0 indicate some postural issues.

\subsubsection{Level and Unlevel $2 \times 10^{\prime}$ Walk}

Clients were asked to walk a level and unlevel $2 \times 10$ foot line as fast as possible. The amount of time (seconds) that it took them to walk from point A to point B and back to point A was recorded. Walking on various surfaces requires participants to recognize and anticipate changes in the walking surface and adapt/prepare their system accordingly (Shumway-Cook \& Woollacott, 2011). The level surface utilized in this study was a smooth concrete floor located indoors, while the unlevel surface was presented as a grassy area located outdoors. Differences in terrain also allow the researchers to determine if the subjects' coordination remains uniform or if it is affected by varying environments. 


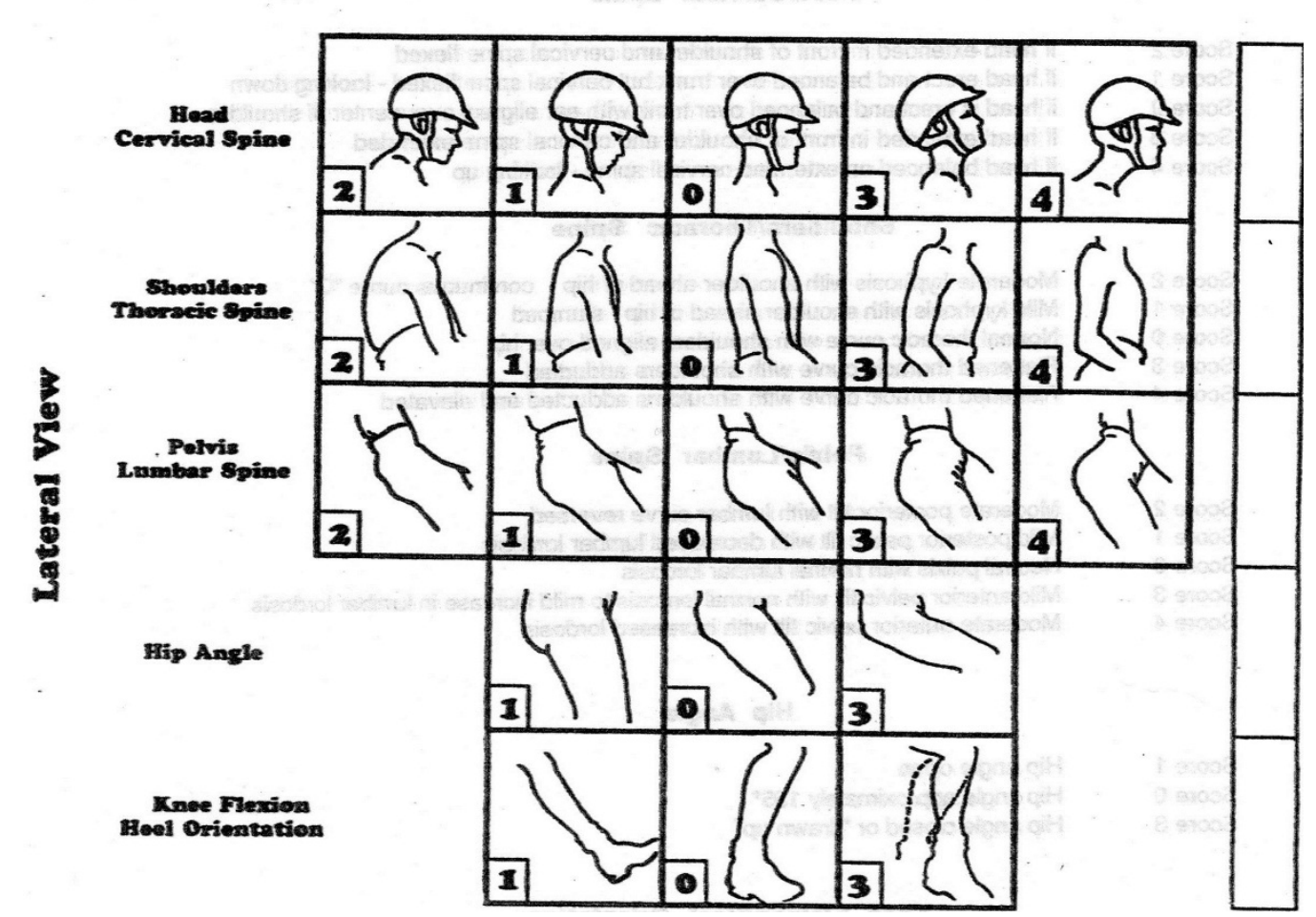

Figure 1. Gainesville riding through equine assisted therapy, designed by linda frease from the university of florida.

\subsubsection{Forward Reach}

Forward reach measures were taken from a standing or sitting position, based on the ability of the subject. Each subject reached their preferred arm out parallel to the floor so the researcher could determine the starting position of the metacarpophalangeal (MCP) joint of the fifth digit. The farthest distance the individual could reach without taking a step was recorded for pre-, and post-time frames. This measure assesses the core strength of the individual by measuring the distance they can reach their arm anteriorly before taking a step forward. Specifically, subjects must have control of their posture when reaching to the extreme limits of their base of support. The development of strength and coordination to complete such tasks typically occurs by one year when sitting (Hadders-Algra, Brogren, \& Forssberg, 1998). A further reach distance suggests stronger core muscle and better postural control (Shumway-Cook \& Woollacott, 2011).

\subsubsection{Physical Therapy Notes}

Along with the physical function measures discussed previously, physical therapy notes including specific goals and improvements for 5 of the subjects were also analyzed to determine changes or improvements in the clients' posture, strength, and coordination. Therapist 1 worked with Subjects 1 - 4 and 8, while Therapist 2 worked with Subjects $5-7$. Only the first therapist provided the researchers with therapy notes for qualitative assessment for the current study. Therapy notes were assessed qualitatively by noting goals for the subjects and improvement on those goals through the course of therapy. In addition, any notes that were related to the study's measurements were also noted.

\subsubsection{Psychological Measures}

Three pediatric quality of life scales were utilized to assess quality of life of the subjects. It has been shown that even in short intervention studies, improvements are recorded which even if not significant have real life benefits 
of the intervention having psychological benefits (Damiano, Arnold, Steele, \& Delp, 2010; Oriel, Marchese, Wagner, Young, \& Miller, 2012). Researchers determined that parent report versions of these questionnaires would yield the most accurate indicators of psychological measures due to the limited level of understanding of the subjects. The PedsQL-Standard Cognitive Functioning Scale and the PedsQL-Standard Pediatric Quality of Life Inventory was given to the parents of all the subjects. The PedsQL-Standard General Well-Being Scale was given only to the parents of the subjects who were between the ages of 8 and 12. Physical therapy notes from the subjects' physical therapist were also analyzed to evaluate notable self-esteem levels. All psychological measures were assessed at two times during the study: pre- and post-.

\subsubsection{PedsQL-Standard Cognitive Functioning Scale (Varni, 1998a, 1998b)}

This particular PedsQL questionnaire was included in the current study because parents of the subjects were able to rate their opinions about their child's cognitive functioning by indicating that their child 0-Never has problems, 1-Almost never has problems, 2-Sometimes has problems, 3-Often has problems, or 4-Almost always has problems with various aspects of cognitive functioning.

\subsubsection{PedsQL-Standard Pediatric Quality of Life Inventory (Varni, 1998a, 1998b)}

A second PedsQL questionnaire that was used for this study was utilized to determine the quality of life of the subject based on four sections: Physical Functioning, Emotional Functioning, Social Functioning, and School Functioning. Again parents were asked to rate their opinions about their child's functioning levels for these categories by indicating that their child 0-Never has problems, 1-Almost never has problems, 2-Sometimes has problems, 3-Often has problems, or 4-Almost always has problems with these specific areas. This questionnaire asked the parents to respond to twenty-three total questions, which were broken down into eight Physical Functioning, five Emotional Functioning, five Social Functioning, and five School Functioning questions.

\subsubsection{PedsQL-Standard General Well-Being Scale (Varni, 1998a, 1998b)}

The final questionnaire that was included in this study was given only to the parents of the children who were between the ages of 8 - 12 years. The purpose of this particular questionnaire was to assess the two sections, general well-being and overall health of the subjects. Parent responses were indicated by 0-Never, 1-Almost never, 2-Sometimes, 3-Often, or 4-Almost always. This questionnaire had seven total questions, six of which were related to general well-being and one that pertained specifically to overall health levels.

\subsubsection{Statistical Analysis}

Results were analyzed with a related samples Wilcoxon Signed Rank test to determine differences between preand post-data (SPSS, v. 21). Significance levels were set at $p<0.05$ and trends were discussed at $p<0.10$.

\section{Results}

\subsection{Physical Function Measures}

\subsubsection{G.R.E.A.T. Postural Scale}

Five of the eight participants ( 63\%) had a lower total score when comparing pre- to post-, while three of the eight participants' scores ( $\sim 37 \%)$ remained constant. A related samples Wilcoxon signed rank test showed statistically significant improvements in posture pre- to post- $(p<0.05)$. It is important to note that the 3 subjects that did not show improvements had the more progressive and severe developmental disorders.

\subsubsection{Level 2 x 10’ Walk}

Six of the seven participants ( $86 \%$ ) decreased the amount of time it took for them to travel the level $2 \times 10$ ' distance, while one of the seven participants ( 14\%) did not decrease their time from pre- to post- (subject 4$)$. A related samples Wilcoxon signed rank test indicates statistically significant decreases in timed walk from pre- to post-test $(p<0.05)$.

\subsubsection{Unlevel $2 \times 10^{\prime}$ Walk}

Three of the six participants (50\%) were able to complete the unlevel walk quicker during post-therapy compared to their performance time pre-therapy. The other three participants (50\%) were unable to decrease the time 
it took for them to travel the unlevel distance pre- to post-. A related samples Wilcoxon signed rank test found no significant findings or trends $(p>0.05)$.

\subsubsection{Forward Reach}

Four of the eight participants (50\%) were able to reach a farther distance before taking a step during their postdata collection compared to their pre-data value. A significant trend between pre- and post- were found from the related samples Wilcoxon signed rank test $(p=0.07)$. It is important to note that 3 subjects improved substantially and they were the subjects with developmental delays (subjects $6-8$ ).

\subsection{Physical Function Improvements from Physical Therapy Notes}

Physical Therapy notes were analyzed for five of the subjects to determine actual improvements that were reported by a licensed physical therapist (Table 2). Four of the five subjects were reported to have increased their endurance levels over hippotherapy sessions as determined by the time spent on horseback before signs of fatigue, and all of the participants were indicated to have improved in postural stability via different mechanisms. Two of the subjects presented pre-therapy with hypertonia, meaning increased muscular tension and a reduced ability to stretch, while three of the subjects started the program with hypotonia, presented by low muscular tone and reduced muscle strength. Both individuals with hypertonia significantly decreased their muscle tone pre- to post-, and two of the three subjects with hypotonia significantly increased their muscle tone by the end of the program. Finally, four of the five participants met their individual goals of ambulation or stair utilization pre- to post-test.

\subsubsection{Psychological Data}

All of the subjects' parents completed the psychological questionnaires prior to the start of the study, but only seven sets of questionnaires were completed post-study. Due to circumstances regarding the reliability of parent data for Subject 7, this subjects' psychological data was removed from the statistical analyses. Subject 3 never returned questionnaires and so this subject was also excluded

\subsubsection{PedsQL-Standard Pediatric Quality of Life Inventory}

A related samples Wilcoxon signed rank test analysis was performed on the data, and there were trends for significant improvements for physical function $(p=0.06)$, emotional function $(p=0.08)$, school function $(p=0.10)$, and total function $(p=0.09)$. The cognitive functioning scale and general well-being scale did not show significant improvements or trends $(p>0.1)$.

Table 2. Results from Analysis of PT Notes, hyper = hypertonia, hypo = hypotonia.

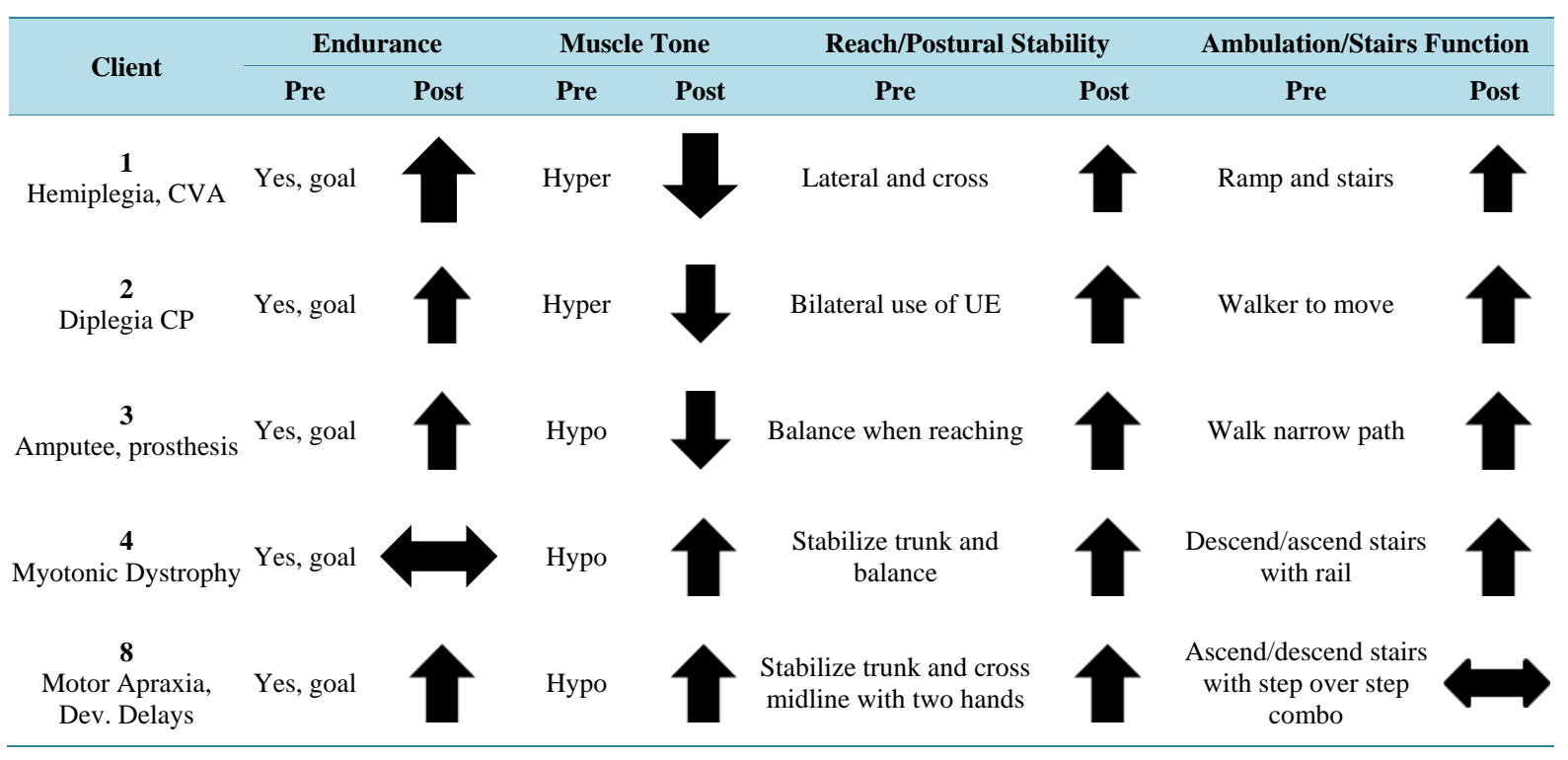




\section{Discussion}

These findings support the utilization of hippotherapy as an effective therapeutic protocol for children with a variety of developmental delays. Until recently, the use of horse therapy has not been seen as a beneficial option for children with developmental delays and motor deficits (Frank, McCloskey, \& Dole, 2011). However, the results of the present study provide evidence through physical function measures, physical therapy notes, and psychological data that hippotherapy can and should be incorporated into these children's therapeutic programs.

Significant improvements were found in three of the physical function measures: Postural Scale, Level $2 \times 10$ ' Walk, and reach test. Improvements in the Postural Scale, as seen by lower total score, suggest that hippotherapy has helped to improve posture and trunk control for most of the clients which also allows them to functionally reach further. This improvement in postural strength and trunk flexibility leading to improved functional reach is a development process (Hadders-Algra, Brogren, \& Forssberg, 1998; Shumway-Cook \& Woollacott, 2011). These results are likely due to the continuous movement of the horse, forcing the participants to constantly correct their trunk positions in order to remain upright. The utilization and engagement of the trunk muscles eventually strengthens those muscles, allowing for better postural control post-therapy (Shurtleff, Standeven, \& Engsberg, 2009). The implications of the improvements in the level walk suggest that the subjects' hippotherapy sessions have improved the subjects' walking coordination in that they are able to travel the same distance in a shorter amount of time. The improvements in the walking coordination are most likely due to improved trunk stability as well as strengthening of the lower extremities. Results of the unlevel walk did not show similar decreased times to travel the distance, indicating that hippotherapy has only been able to help these subjects perform better in normal conditions (i.e. level ground), but it is likely given practice and the organization of control that these improvements will eventually translate to uneven ground when predictability of control decreases (Shumway-Cook \&Woollacott, 2011).

In addition, the Physical Functioning, emotional functioning, school functioning, and total functioning sections of the PedsQL-Standard Pediatric Quality of Life Inventory showed a trend towards improved function suggesting the benefits of hippotherapy may continue to be seen functionally over time. While the Forward Reach test only showed a trend toward improvement, it was important to note the subjects that saw improvement. These subjects had developmental delays primarily and were not dealing with known progressive disorders or severe restriction in lower body movement. Most of the subjects have coordination and postural control difficulties initially, and those that were more severe were not willing to move themselves away from their center of gravity any more than were required. It is likely that for our population (young children with postural control difficulties) this test challenged them both psychologically related to comfort level and physically.

Though the researchers expected to see improvements in all measures, there are several factors that would explain why only three of the physical function measures showed significant improvements or trends. As this study is specifically looking at pediatric populations, the motivation and level of understanding of the participants may have interfered in obtaining best performances especially in the high temperatures of the humid summer. It was clear that participants were much more engaged on the horse than on our tests. Researchers demonstrated the measures for the participants repeatedly and encouraged the participants to give their best effort in all measures, but despite the researchers' attempts, the participants did not always comply. Perhaps a larger sample size would also allow for significant improvements to be seen, as this study only utilized data from eight subjects. However, because of the novelty of this treatment, it is difficult to find a large pool of subjects and therapists to pull from. We were fortunate to have the cooperation we had from the therapists, the subjects, and their families and the improvements for individuals was profound.

In order to account for levels of exhaustion, the researchers had the participants complete the physical function measures prior to their therapy on the days in which the participants were tested. However, due to varying therapy sessions and schedules, all testing could not be completed at the same time of day. Baseline measures were taken before noon at the subjects' respective therapy time because the subjects were not attending school during the summer months. Post-measures were taken in the late afternoon because therapy sessions were altered due to the participants' return to school in the fall. These variables could not be accounted for within this study, and it is possible that improvements in several of the measures could not be verified due to these factors.

Another confounding variable in this study was the number of hippotherapy sessions that the participants actually attended. The physical therapists arranged to have all of their clients attend a hippotherapy session on Wednesdays each week, with the exception of planned family vacations. One time per week is standard of care. 
Due to varying circumstances, not all participants actually received the same amount of hippotherapy sessions, a number of sessions missed were not recorded but they never exceeded two.

Despite the confounding factors, the present study was able to confirm physical function and psychological benefits for the majority of our participants. Similar implications have been found in other recent hippotherapy/therapeutic riding therapies specifically related to postural and motor control (Silkwood-Sherer et al., 2012; Zadnikar \& Kastrin, 2011). The first known meta-analysis related to this therapy was completed to assess the effects of hippotherapy programs on postural control or balance in children with varying forms of Cerebral Palsy (Zadnikar \& Kastrin, 2011). An analysis of eight individual studies indicated that hippotherapy provided a therapeutic benefit in children with Cerebral Palsy, specifically in improved postural control and balance, but also in other motor functions that are needed to complete activities of daily living (Zadnikar \& Kastrin, 2011). Thus, this study is one of the first to look at more global developmental delays with positive results. The ultimate goal of a hippotherapy program is to improve the initial motor deficits in the individuals, thereby allowing them to gain motor functions and learn to appropriately maneuver themselves in a variety of environments (Frank, McCloskey, \& Dole, 2011).

Overall, this study shows improvements in function for children with developmental delays when participating in hippotherapy as part of their therapy regime. Hippotherapy is a new therapeutic tool that is not often covered by insurance policies due to the lack of data on the efficacy and feasibility of this treatment. Our participants were motivated, engaged, and happy during their 45 minute hippotherapy sessions and made tremendous improvements given the number of confounding factors related to research in the heat of the summer with a population of children that have a lot of factors affecting their ability to participate. This data is so encouraging that the power of animal assisted therapy, specifically hippotherapy, can show improvements in motor function which was targeted by the therapist along with psychological improvements that will continue to benefit all aspects of their lives.

\section{Acknowledgements}

We would like to thank the therapists, parents and participants of this study particularly the physical therapists at Therapy Source, Susan Pearce and Susan Adams.

\section{References}

Bass, M., Duchowny, C., \& Llabre, M. (2009). The effect of therapeutic horseback riding on social functioning in children with autism. Journal of Autism and Developmental Disorders, 39, 1261-1267. http://dx.doi.org/10.1007/s10803-009-0734-3

Bertoti, D. B. (1998). Effect of Therapeutic Horseback Riding on Posture in Children with Cerebral Palsy. Physical Therapy, 68, 1505-1512.

Boyle, C. A., Boulet, S., Schieve, L. A., Cohen, R. A., Blumber, S. J., Yeargin-Allsopp, M., Visser, S., \& Kogan, M. D. (2011). Trends in the Prevalence of Developmental Disabilities in US Children, 1997-2008. Peadiatrics, 134, 1034-1042. http://dx.doi.org/10.1542/peds.2010-2989

Cantu, C. O. (2005) Hippotherapy: Facilitating Occupational Performance. The Exceptional Parent, 35, 51-53.

Damiano, D. L., Arnold, A. S., Steele, K. M., \& Delp, S. L. (2010). Can Strength Training Predictably Improve Gait Kinematics? A Pilot Study on the Effects of Hip and Knee Extensor Strengthening on Lower-Extremity Alignment in Cerebral Palsy. Physical Therapy, 90, 269-279. http://dx.doi.org/10.2522/ptj.20090062

Frank, A., McCloskey, S., \& Dole, R. (2011). Effect of Hippotherapy on Perceived Self-Competence and Participation in a Child with Cerebral Palsy. Pediatric Physical Therapy, 23, 301-308. http://dx.doi.org/10.1097/PEP.0b013e318227caac

Frease, L. (1996). The G.R.E.A.T. Postural Scale. Gainesville, FL: University of Florida.

Hadders-Algra, M., Brogren, E., \& Forssberg, H. (1998). Development of Postural Control—Differences between Ventral and Dorsal Muscles? Neuroscience and Biobehavioral Reviews, 22, 501-506. http://dx.doi.org/10.1016/S0149-7634(97)00036-5

Hammer, A., Nilsagard, Y., Forsberg, A., Pepa, H., Skargren, E., \& Oberg, B. (2005). Evaluation of Therapeutic Riding (Sweden)/Hippotherapy (United States). A Single-Subject Experimental Design Study Replicated in Eleven Patients with Multiple Sclerosis. Physiotherapy Theory and Practice, 21, 51-77. http://dx.doi.org/10.1080/09593980590911525

Macauley, B. L., \& Guiterrez, K. M. (2004). The Effectiveness of Hippotherapy for Children with Language-Learning Disabilities. Communication Disorders Quarterly, 25, 205-217. http://dx.doi.org/10.1177/15257401040250040501 
McGee, M. C., \& Reese, N. B. (2009). Immediate Effects of a Hippotherapy Session on Gait Parameters in Children with Spastic Cerebral Palsy. Pediatric Physical Therapy, 21, 212-218. http://dx.doi.org/10.1097/PEP.0b013e3181a39532

Meregillano, G. (2004). Hippotherapy. Physical Medicine and Rehabilitation Clinics of North America, 15, 843-854. http://dx.doi.org/10.1016/j.pmr.2004.02.002

Molloy, C., Dietrich, K., \& Bhattacharya, A. (2003). Postural Stability in Children with Autism Spectrum Disorder. Journal of Autism and Developmental Disorders, 33, 643-652. http://dx.doi.org/10.1023/B:JADD.0000006001.00667.4c

Oriel, K. N., Marchese, V. G., Shirk, A., Wagner, L., Young, E., \& Miller, L. (2012). The Psychosocial Benefits of an Inclusive Community-Based Aquatics Program. Pediatric Physical Therapy, 24, 361-367. http://dx.doi.org/10.1097/PEP.0b013e3182691792

Rosenberg, S. A., Zhang, D., \& Robinson, C. C. (2008). Prevalence of Developmental Delays and Participation in Early Intervention Services for Young Children. Pediatrics, 121, e1503-e1509.

Shumway-Cook, A., \& Woollacott, M. (2011). Motor Control: Translating Research into Clinical Practice (4th ed.). Philadelphia, PA: Lippincott Williams \& Wilkins.

Shurtleff, T., Standeven, J., \& Engsberg, J. (2009). Changes in Dynamic Trunk/Head Stability and Functional Reach after Hippotherapy. Archives of Physical Medicine and Rehabilitation, 90, 1185-1195.

http://dx.doi.org/10.1016/j.apmr.2009.01.026

Silkwood-Sherer, D. J., Killian, C. B., Long, T. M., \& Martin, K. S. (2012). Hippotherapy-An Intervention to Habilitate Balance Deficits in Children with Movement Disorders: A Clinical Trial. Physical Therapy, 92, 707-717. http://dx.doi.org/10.2522/ptj.20110081

Sterba, J., \& Warner, M. (2007). Does Horseback Riding Therapy of Therapist-Directed Hippotherapy Rehabilitate Children with Cerebral Palsy? Developmental Medicine and Child Neurology, 49, 68-73. http://dx.doi.org/10.1017/S0012162207000175.x

Taylor, R., Kielhofner, G., Smith, C., Butler, S., Cahill, S., Ciukaj, M., \& Gehman, M. (2009). Volitional Change in Children with Autism: A Single-Case Design Study of the Impact of Hippotherapy on Motivation. Occupational Therapy in Mental Health, 25, 192-200. http://dx.doi.org/10.1080/01642120902859287

Varni, J. (1998a). PedsQL Parent Proxy Report (Ages 5 - 7). PedsQL ${ }^{T M}$.

Varni, J. (1998b). PedsQL Parent Proxy Report (Ages 8 - 12). PedsQL ${ }^{T M}$.

Winchester, P., Kendall, K., Peters, H., Sears, N., \& Winkley, T. (2002). The Effect of Therapeutic Horseback Riding on Gross Motor Function and Gait Speed in Children Who Are Developmentally Delayed. Physical and Occupational Therapy in Pediatrics, 22, 37-50.

Zadnikar, M., \& Kastrin, A. (2011). Effects of Hippotherapy and Therapeutic Horseback Riding on Postural Control or Balance in Children with Cerebral Palsy: A Meta-Analysis. Developmental Medicine \& Child Neurology, 53, 684-691. http://dx.doi.org/10.1111/j.1469-8749.2011.03951.x 